\title{
Kernos
}

Revue internationale et pluridisciplinaire de religion grecque antique

3 | 1990

Varia

\section{Der Seher Mopsos auf den Münzen der Stadt Mallos}

\section{Dieter Metzler}

\section{OpenEdition \\ Journals}

\section{Édition électronique}

URL : http://journals.openedition.org/kernos/992

DOI : $10.4000 /$ kernos.992

ISSN : 2034-7871

\section{Éditeur}

Centre international d'étude de la religion grecque antique

\section{Édition imprimée}

Date de publication : 1 janvier 1990

ISSN : 0776-3824

\section{Référence électronique}

Dieter Metzler, « Der Seher Mopsos auf den Münzen der Stadt Mallos », Kernos [En ligne], 3 | 1990, mis en ligne le 19 avril 2011, consulté le 30 avril 2019. URL : http://journals.openedition.org/kernos/992 ; DOI : $10.4000 /$ kernos.992 


\section{DER SEHER MOPSOS AUF DEN MÜNZEN DER STADT MALLOS*}

Dem Andenken an Hayo Vierck gewidmet.

Die Münzbilder griechischer Städte Kleinasiens verweisen gelegentlich auf die Stadtgründer ${ }^{1}$. Mopsos, als Gründer in mehreren Städten Lykiens, Pamphyliens und Kilikiens ${ }^{2}$ verehrt, konnte von $\mathrm{L}$. Robert auf Silberdrachmen von Aspendos aus dem 5. Jhd. v. Chr. identifiziert werden. Wie er dort, einem lokalen Mythos entsprechend, als Reiter auf der Eberjagd der Artemis zugeordnet ist, denn ihr gilt das Opfer des Ebers, der auf dem Münzrevers dargestellt ist ${ }^{3}$, so möchte ich in dem so beschriebenen "Geflügelten Genius im Knielauf n.r. einen mit Stern geschmückten Diskus haltend" ${ }^{4}$ auf den Silberstateren der kilikischen Stadt Mallos vom Ende des 5. und Anfang des 4. Jdhs. v. Chr. (Abb. 1) einen der beiden Gründer ${ }^{5}$ dieser Stadt, nämlich ebenfalls

* Anregungen und wichtige Literaturhinweise - besonders in Fragen des Schamanismus - verdanke ich den fruchtbaren Diskussionen mit meinem münsteraner Kollegen Hayo Vierck. Er starb, kaum fünfzig Jahre alt, am 16.3.1989 - am selben Tage, an dem ich diesen Vortrag halten durfte.

1 J.H.M. STRUBBe, Gründer kleinasiatischer Städte : Fiktion und Realität, in AncSoc, 15-17 (1984-1986), p. 253-304; P.R. FRANKE, Kleinasien zur Römerzeit. Griechisches Leben im Spiegel der Münzen, München, 1968, p. 18, 28 sq.

2 D. MAGIE, Roman rule in Asia Minor, Princeton, 1950, p. 1132 sq.; O. IMMISCH, Klaros, in Fleckeisens Jhbb. f. class. Phil., Suppl, 17, Leipzig, 1890, p. 160-166, bes. 163.

3 L. RoBer', Monnaies et divinités d'Aspendos, in Hellenica, Paris, 1977, p. 177 sq., pl. 37-40.

4 Münzen und Medaillen AG Basel, Liste 506, Januar 1988, Text zu Abb. 33. Durch diese Abbildung des relativ häufigen Münztyps kam ich auf meine Deutung. Der Leitung der MMAG danke ich für die freundlich gewährte Reproduktionserlaubnis. - Literatur : E. BABELON, Traité des monnaies grecques et romaines, II, Paris, 1910, n 1390-1404, pl. 137; B.V. HEAD, Historia Numorum, London, $1911^{2}$, p. 723, fig. 320; C.M. KRAAY, Archaic and classical Greek coins, London, 1976, $\mathrm{n}^{\circ}$ 1020-1022; SNG Switzerland I, bearb. v. E. LeVANTE, Cilicia, Bern, 1986, $\mathrm{n}^{\circ}$ 124-136 (P. Ilisch danke ich für diesen Hinweis). - Eine neuere Monographie gibt es seit F. IMHOOF-BLUMER, Abri $\beta$ der mallotischen Münzprägung, in Annuaire Numismatique, 7 (1883) nicht, wie mir freundlicherweise S. ScHULTZ (Münzkabinett Berlin DDR) mitteilte. Frau ScHULTz' numismatische Bibliographie zu Kilikien wird in Chiron, 18 (1988) erscheinen.

5 STRAB., XIV, 5, 16 (675). Zur Geschichte der Stadt und zur Verehrung ihrer Gründer vgl. R. ZIEGLER, Städtisches Prestige und kaiserliche Politik. Studien 
Mopsos, den Sohn Apolls erkennen. Der Schwan auf dem Münzrevers ist das Tier Apolls par excellence ${ }^{6}$, dessen Sehergabe (mantiké) es seinen eigenen Tod vorankündigen läßt ${ }^{7}$.

Flügel sind in der etruskischen und griechischen Kunst ein charakteristisches Merkmal für die Darstellung von Sehern. Darauf hat mit Bild- und Schriftquellen P. Wolters 1927 hingewiesen $^{8}$. Sein point de départ ist der bekannte etruskische Bronzespiegel (Abb. 2) mit Kalchas bei der Leberschau ${ }^{9}$. An die Stelle der älteren, poetischemblematischen Interpretation des Sehers, "dessen prophetischer Schwung sich durch Beflügelung kundgibt» ${ }^{10}$ setzte er den Hinweis auf die mannigfaltigen Beispiele für beflügelte Menschen, Heroen, Dämonen und Götter in der Bilderwelt der Griechen ${ }^{11}$. In unserem Zusammenhang sind darunter von besonderem Interesse der geflügelte Argonauten-Seher auf der ficoronischen Ciste ${ }^{12}$ - möglischerweise sogar ebenfalls ein Mopsos ${ }^{13}$, Namensvetter des Gründers von Mallos -

zum Festwesen in Ostkilikien im 2. und 3. Jhd. n. Chr., Düsseldorf, 1985, p. 9497.

6 W. RICHTER, art. Schwan, in Kleine Pauly, V (1975), col. 43. Kyknos = Schwan als Sohn Apolls : K. KERÉnYI, Die Mythologie der Griechen, II, München, 1966, p. 270. Cf. auch D. METZLER, Zum Schamanismus in Griechenland, in Antidoron. Festschrift für J. Thimme, Karlsruhe, 1983, p. 75. - Aphrodite/Venus wird mit dem Schwan in römischer Zeit verbunden (RICHTER, art. cit.) anders HEAD, art. cit. Allerdings werden Schwan und Gans der Aphrodite auch verwechselt (RICHTER, art. Gans, in Kl. Pauly, II (1967), col. 694, zu der «weißgrundigen Schale des Pistoxenos-Malers, London D $2=$ $A R V^{2}, 862,22$.

7 Plat., Phaidr., 84e.

8 P. Wolters, Der geflügelte Seher, München, 1928 (Sitz. ber. Bayer. Akad. Wiss.), p. 1.

9 Vatikan, Museo Gregor. Etrusc. HELBIG $4, \mathrm{n}^{\circ} 724$ (T. DoHrN). Civilta degli Etruschi = Ausstellungskatalog Florenz, 1985, p. 355 (Farbtafel).

10 E. GERHARD, Etruskische Spiegel, III, Berlin, 1863, p. 212, pl. 223.

11 Cf. H. KeNNER, Flügelfrau und Flügeldämon, in ÖJH, 31 (1939), p. 81-95. C. ISLER-KERÉNYI, Nike. Der Typus der laufenden Flügelfrau in archaischer Zeit, Zürich-Stuttgart, 1969.

12 Rom, Villa Giulia. Helbig ${ }^{4}$, n 2976 (T. DOHRN). Wolters, op. cit, p. 15 sq. T. DoHrn, Die ficoronische Ciste, Berlin, 1972.

13 Gegen die klassische Interpretation des Geflügelten als Boreas sprach sich schon Wolters aus. Ich sehe im Hüftmantel sowie im Gestus des aufgestützten Beines - wie bei Kalchas auf dem Spiegel - und der Hand am Kinn - wie beim alten Seher des Olympia-Giebels nichts für Boreas charakteristisches, folge also Wolters' Vorschlag, einen der Argonauten-Seher zu erkennen 
und der nach Pausanias fliegend dargestellte Mousaios in der Pinakothek der Akropolis ${ }^{14}$. Mit den erwähnten beiden etruskischen Seher-Darstellungen hat die geflügelte Gestalt auf dem Haupttypus der Münzen von Mallos die Form der Bekleidung gemeinsam. Es handelt sich jeweils um einen Hüftmantel, der den Oberkörper frei läßt ${ }^{15}$. In Mallos gibt es daneben zwei Varianten, die ebenfalls hierher gehören, da auch sie die männliche geflügelte Gestalt mit dem weiter unten zu besprechenden «Diskus» in den Händen zeigen. Die erste zeigt eine jugendliche Gestalt im Knielauf nach links eilend unbekleidet ${ }^{16}$, die zweite frontal einen bärtigen, doppelköpfigen Mann mit vier Flügeln ${ }^{17}$, dessen Unterkörper nicht erkennbar hinter der Protome eines Stiers ${ }^{18}$ verdeckt ist. Die Münzen der Vierflügeligen Gestalt haben genau wie die mit der bekleideten zweiflügeligen jeweils auf der Rückseite den Schwan - im Typus vergleichbar den Rückseiten der von Theodotos signierten Apollon-Tetradrachemn von Klazomenai ${ }^{19}$.

Ich gestehe, daß - wie mir scheint notwendige - Einbeziehung der vierflügeligen janiformen Gestalt meinen Deutungsvorschlag in gewisser Weise belastet. Doppelköpfige Seher-Darstellungen kenne ich bisher nicht, es sei denn, man dürfte sich bei den männlich-weiblichen janiformen Köpfen der Münzen von Tenedos ${ }^{20}$ an den Seher Teiresias

- möglicherweise also Mopsos, Sohn des Ampyx und der Chloris, Lapith aus Thessalien (Roscher, $R E$ und $E A A$, art. Mopsos jeweils unter Nr. 1).

14 PAUS., I, 22, 7. WOLTERS, op. cit., p. 8 sq.

15 HEAD, op. cit., pl. 320; KrAAY, op. cit., n 1022.

16 KRAAY, op. cit., $n^{\circ}$ 1020. Auf dem Revers hier Hermes einen Widder reitend ein Unikum. Meist hat auch dieser Typus auf dem Revers den Schwan.

17 KRAAY, op. cit., n' $1021=$ G. MACDONALD, Catalogue of Greek coins in the Hunterian Collection, II, Glasgow, 1899, p. 536, $\mathrm{n}^{\circ}$ 1-2, pl. 59, 11-12. BABELON, op. cit., $\mathrm{n}^{\circ} 1390$, pl. 137, 16-17.

18 KRAAY, op. cit., p. 285. Der Stier kann den Gott des Flusses Pyramos bei Mallos darstellen. Die Deutung der abgeriebene Oberflache des Diskus auf den Münzen im Hunter Coin Cabinet als Bukranion (zuletzt KRAAY, op. cit.) halte ich für arbiträr, ebenso die daran anknüpfende Deutung als «Kronos ?» bei HEAD.

19 HEAD, op. cit., p. 568, pl. 293; KRAAY, op. cit., $\mathrm{n}^{\circ} 929$.

20 HEAD, op. cit., p. 550, pl. 288. Die alte Deutung des Doppelkopfes der TenedosMünzen als Zeus und Hera ist nicht begründbar, geläufiger ist die mit Tennes und Hemithea, zumal Konon (26 F 1, 28 J. - aus Photios) im selben Kontext auch die Doppelaxt - wohl geradezu nach dem Revers der Tenedos-Münzen erwähnt. 
erinnert fühlen, von dessen Geschlechtswandel ${ }^{21}$ die antiken Quellen erzählen. Leider ist auch das hier zu berührende Problem der Dopplherme ${ }^{22}$ keineswegs soweit erschlossen, daß von dort her Lösungen zu erwarten wären. Deshalb sei ein Blick auf eine nur scheinbar weit entfernt liegende mögliche Parallele zum geflügelten Seher gestattet : Doppelköpfig ist der Adler, in dessen Gestalt sich die sibirischen Ostjaken am Jenissei ihren ersten Schamanen vorstellen ${ }^{23}$. Die zunächst befremdliche Distanz verkürzt sich, zieht man die von F. Altheim ${ }^{24}$ entdeckte Entsprechung der Tierkampfszenen von Elch und Vielfraß (gulo gulo) auf einem Filzteppich von Noin Ula in der Mongolei und auf einer Stele aus dem nicht weit von Mallos entfernten - syrischen Tell Halaf in Betracht. Der hier dargestellte Kampf von Elch und Vielfraß ist seinerseits nun wiederum im Landnahmemythos einer ebenfalls ostjakischen 25 Gruppe belegt.

Während P. Wolters den geflügelten Seher noch im Vergleich zum Bild der Flügel des Sängers ${ }^{26}$ sowie anderer griechischer Vorstellungen vom Fliegen der Götter sehen wollte und die Berichte über den Skythen Abaris eher beiläufig als "eine wunderliche Überlieferung» 27 heranziehen konnte, ist es seit dem bahnbrechenden Aufsatz von K. Meuli durchaus üblich, auch Vergleiche mit dem eurasiatischen Schamanismus zum Verständnis mancher Elemente

21 APOllod., Epitome, 5, 4. KerÉnYi, op. cit., p. 85. Cf. K. MEULI, Scythia, in Hermes, 70 (1935), p. 121-176, bes. 127 sq.; H. FindeISEN, Schamanentum, Stuttgart, 1957, p. 140 sq.

22 J. MARCadé, Hermès doubles, in $B C H, 76$ (1952), p. 596-624; S. SEILER, Beobachtungen an Doppelhermen, Diss. Hamburg, 1969, p. 45 sq.

23 B.D. SHImKIN, A sketch of the Ket or Yenissei Ostyak, in Ethnos, 4 (1939), p. 147-176, bes. 160 sq.; J. HALIFAX, Schamanen, Frankfurt, 1981, p. 23. - Zum Doppeladler in Ur III, bei den Hethitern, in Taxila und in Turfan: E. KoRnemann, Adler und Doppeladler im Wappen des Deutschen Reiches. Zur Vorgeschichte des Doppeladlers = Gestalten und Reiche, Wiesbaden, 1943, p. 402-415. Zur Verdoppelung des Flügelpaares auf der Mallos-Münze, cf. die Verdoppelung der Elchfüße auf acht im Mythos der Ostjaken (ALTHEIM, cf. infra n. 24, n. 203) und ähnliche Erscheinungen im germanischen Mythos.

24 F. AltheIM, Weltgeschichte Asiens im griechischen Zeitalter, II, Halle, 1948, p. 200-202; A. AlFöLDI, Die Struktur des voretruskischen Römerstaates, Heidelberg, 1974, p. 40 sq., pl. 4, 1-2.

25 AlTHEIM, op. cit., p. 201.

26 WOLTERs, op. cit., p. 9 sq.

27 WOLTERS, op. cit., p. 10. 
der griechischen Religion heranzuziehen ${ }^{28}$. Der Schamanenflug ${ }^{29}$ und die damit meist verbundene - partielle oder umfassende Tierverwandlung 30 bilden in diesem Kontext natürlich ganz besonders wichtige und naturgemäß bekannte Vorstellungskomplexe. Da der dem erwähnten Abaris vergleichbare Aristeas auch in Unteritalien verehrt $^{31}$ worden ist, scheint es mir erwägenswert, ihn oder auch Orpheus in den Darstellungen von männlichen Köpfen mit phrygischer = skythischer Mütze zwischen Flügeln ${ }^{32}$ als einen geflügelten Seher zu erkennen. Dem sepulkralen Bereich zugehörig hätten die Dargestellten hier die Funktion von Seelengeleitern. Schamanen haben auch diese Funktion.

28 Cf. supra n. 21. Cf. auch E.R. DodDs, Die Griechen und das Irrationale, Darmstadt, 1970 (amerikan. 1951), p. 72-91; W. BURKERT, ГOHL. Zum griechischen Schamanismus, in RhM, 105 (1962), p. 36-55; METZLER, art. cit. n. 6.

29 M. ELIADE, Schamanismus und archaische Ekstasetechnik, Frankfurt, 1975 (französ. 1951), p. 157-159 et passim; ID., Der magische Flug, in Antaios, 1 (1960), p. 1-11; G.M. Bongard-LEvin - E.A. GRANTOVSKIJ, De la Scythie à l'Inde, Paris, 1981 (russ. 1974), p. 83-88, bes. 87.

30 R. MERz, Die numinose Mischgestalt. Methodenkritische Untersuchungen zu tier-menschlichen Erscheinungen Altägyptens, der Eiszeit und der Aranda in Australien, Berlin-New York, 1978, p. 219 sq. (griechische Götter), 233 sq. (Schamanismus). Zur "Ornithophanie» hatte Hayo VIERCK in seiner Habilitationsschrift Wieland der Schmied... und Schamane?, 1987, wichtige Beobachtungen beigetragen. Einen der Ausgangspunkte seiner weitgespannten Untersuchungen bildeten natürlich so bekannte Darstellungen wie die Wieland-Saga auf dem Kasten von Auzon cf. K. HAUCK, in Reallexikon german. Altertumskunde, I2 (1973), p. 514-522, und besonders Wieland mit dem Vogel auf dem gotländischen Bildstein Ardre VIII (ebd. 398, p. 71, pl. 32 [W. HoLMQVIST]). Dieser Zusammenstellung analog scheint mir auch der Schwan bei dem Sänger/Schamanen Eumolpos auf dem Skyphos des Makron (London, Brit. Mus. E 140. ARV2, 459, 3- nach Wolters, op. cit., p. 9) deutbar zu sein.

31 MeUli, op. cit., p. 153 sq.; J. WIESNER, Studien zu dem Arimaspenmotiv auf Tarentiner Sarkophagen, in JdI, 78 (1963), p. 200-217, bes. 205.

32 K. SChauenBurg, Zu unteritalischen Situlen, in AA (1981), p. 462-488, bes. 464 sq. Abb. 5 (Gnathia-Situla in Hannover, Kestner-Museum) und 468, n. 20 (Situla in Ruvo, Coll. Jatta 1372). - Zu den Sternsymbolen in Gestalt von drei Punkten auf der Mütze des Kopfes auf der Situla in Hannover vgl. die Sternsymbole der Indus-Kultur (A. PARPOLA, The sky-garment, Helsinki, 1985, p. 55 sq.), die auch die Erklärung für das sogenannte «Chintamani»-Motiv auf asiatischen Textilien (M. LIPTON, Les tapis-tigres du Tibet, Bruxelles, 1988, p. 164-168) bieten können. 
Andeuten möchte ich nur das Problem der Deutung des Knielaufschemas. Auf einer ersten Ebene des Verständnisses sind die angewinkelten Beine des Flügelwesens natürlich Zeichen für die Schnelligkeit seiner Bewegung ${ }^{33}$. Unter dem Aspekt des Einknickens der Beine ist darüber hinaus auch an Tänze mit Hink-, Stolper- oder Schleifschritten und dem jeweils darauf folgenden Wiederaufrichten zu denken, die im kriegerischen ${ }^{34}$, dionysischen ${ }^{35}$ und sepulkralen ${ }^{36}$ Kontexte als rituelle Vorwegnahme von Auferstehung und Weiterleben getanzt werden.

Die runde Scheibe, die der geflügelte Mann auf den Mallos-Münzen mit beiden Händen vor seinem Körper hält, weist ebenfalls in schamanistische Bereiche. Sie zeigt auf den meisten Exemplaren eine achtstrahlige Rosette, die auf dem Hintergrund der Scheibe im Alten Orient die Sonne symbolisiert ${ }^{37}$. Doch halte ich es für verfehlt, deswegen auf die scheinbare Analogie der ebenfalls altorientalischen Flügelsonne hinzuweisen ${ }^{38}$, denn erstens ist dabei die männliche Gestalt, falls dargestellt, auf der Scheibe bzw. im Ring zwischen den Flügeln dargestellt, das Verhältnis von menschlicher Gestalt zur geflügelten Scheibe also genau umgekehrt und zweitens begegnet genau dieses Bildsymbol in seiner spezifisch iranischen Bedeutung auf den

33 H. WIEgARTz, Zur Deutung der "Waffenläufer-Stele», in Marburger Winckelmannsprogr., 1964, p. 46-64, bes. 50 sq.; P. CALMEYER, art. Knielauf, in Reallex. Assyriologie; D. METZLER, Einflu $\beta$ der Pantomime auf die Vasenbilder des 6. und 5. Jhds. v. Chr., in Images et société en Grèce ancienne, Lausanne, 1984 (= Cahiers d'Archéologie romande, 36 [1987]), p. 73-77, bes. 75)

34 Cf. K. SiтTL, Die Gebärden der Griechen und Römer, Leipzig, 1890, n. 2; F. SOKоLICEK, Der Hinkende im brauchtümlichen Spiel, in Festschrift für O. Höfler, Wien, 1968, p. 423-432, zur semantischen Polyvalenz des HinkeGestus.

35 K. LATTE, Askoliasmos, in Hermes, 85 (1957), p. 385-391; M. Detienne, Dionysos à ciel ouvert, Paris, 1986, p. 73-79.

36 Th. GASTER, Myth, legend and custom in the Old Testament, London, 1969, $\$ 129$ a zu 1. Sam. 15, 32. Cf. auch G. LÜLING, Sprache und archaisches Denken, Erlangen, 1985, p. 75.

37 Cf. zB. Stele aus Nimrud, W. ORthmann, Der Alte Orient, in PropyläenKunstgeschichte, Berlin, 1975, pl. 197, oder die Bronze-Tafel des Königs Marduk-apla-iddine (J. OATEs, Babylon, Bergisch Gladbach, 1983 [engl. 1979], pl. 74).

38 KRAAY, op. cit., p. 285. Cf. R. MAYER-OPIFICIUS, Die geflügelte Sonnenscheibe ein Jahrtausende altes Motiv, in Antidoron. Festschrift für J. Thimme, Karlsruhe, 1983, p. 19-24. 
unter Tiridates geprägten Münzen nicht nur in Mallos, sondern auch in anderen kilikischen Städten, nämlich in Soloi, Tarsos und Issos ${ }^{39}$.

Im Zusammenhang mit dem schamanistischen Seher scheint mir die Sonnenscheibe eher an Branchos ${ }^{40}$, den Stifter des Apollo-Orakels der Branchiden von Milet - Klaros benachbart, wo Mopsos den Kalchas im Schamanen-Kampf ${ }^{41}$ besiegte - zu erinnern, in dem O. von Vacano ${ }^{42}$ mit Recht einen ursprünglich "südrussischen Schamanen" vermutete. Branchos, der "Brüller» 43 wird geboren, nachdem seine Mutter im Traum die Sonne verschluckt hatte ${ }^{44}$.

Als Sonnenscheiben sind nun nicht nur die entsprechenden bronzezeitlichen Goldbleche Nordeuropas gedeutet worden ${ }^{45}$, als solche ist auch wohl die Goldscheibe auf dem Bauch des Toten aus dem Herrschergrabe 43 in Warna (Bulgarien) vom Ende des 5. Jahrtausends zu verstehen ${ }^{46}$. Daß aus dieser Perspektive auch die ursprüngliche Bedeutung der besonders in zahlreichen Grabfunden

39 LEVANTE, op. cit., $\mathrm{n}^{\circ} 49,62,148,175-177$.

$40 R E$, art. Branchos.

41 STRAB., XIV, 1, 27 (642-643). IMMISCH, art. cit.

42 O.W. v. VACANO, Im Zeichen der Sphinx, Stuttgart, 1952, p. 90.

43 Zum häufig nachgewiesenen ekstatischen Brüllen des Schamanen vgl. auch die Stellen der sogenannten Mithras-Liturgie, wo zum Zwecke des Apathanatismos (v. 747) die Sonne unter intensivem Brüllen (mykôma) herbeigezwungen wird (Papyri Graecae Magicae, hrsg. v. K. PREISENDANz A. HENRICHS, I, Stuttgart, $1973^{2}$, v. 658, 705, 712). Zum Getöse der Sonne : A. SCHEIBER, in AAntHung, 19 (1971), p. 395.

44 KonoN, Narr., 33, 3 = 26 F 1, 33, 3 J. Vgl. eine Eskimo-Schamanin, die ihre Befähigung durch eine in ihren Leib eingedrungene Feuerkugel erfuhr und ebenfalls einen Schamanen gebar (ELIADE, op. cit., p. 31 sq.).

45 W. MENGHIN - P. SchaUer, Der Goldkegel von Ezelsdorf. Kultgerät der späten Bronzezeit, Nürnberg-Stuttgart, 1982, p. 17, 44, 50, besonders wichtig dort die Terrakotta-Nachbildung einer Goldscheibe aus einem Grabfund (Fig. 23), da es bei ihr nicht um den materiellen Schmuckwert, sondern um eine höhere Bedeutung geht.

46 Das erste Gold der Menschheit, Ausstellungskatalog, hrsg. v. G. BIEGEL, Freiburg, 1986, p. 30, $\mathrm{n}^{\circ} 311 ; 137$. Die Goldscheibe mit einem Durchmesser von $9,1 \mathrm{~cm}$ war nach der Lage kleinerer Goldknöpfe zu urteilen an einem Kreuzband über der Brust befestigt. Zusammen mit dem goldverzierten Bogen und der goldenen Penishülle scheint sie die Bedeutung einer Herrscherinsignie zu haben. In der Größe und der Tragweise am Körper entspricht sie den kurioserweise als «Herzpanzer» bezeichneten Scheiben, die die bekannte italische Grabfigur des Kriegers von Capestrano und ein entsprechender Skelettfund aus Alfedena zeigen (H. KäHLER, Rom und seine Welt, München, 1960, pl. 5, fig. 16-17). 
(Abb. 3$)^{47}$ zwischen dem Wolgagebiet und Japan verbreiteten 48 chinesischen Spiegel - ihre Reihe beginnt um 2000 v. Chr. mit einem sehr westlich anmutenden Exemplar der Qijia-Kultur aus Qinghai ${ }^{49}$ zu erfassen ist, hat $O$. Münsterberg schon 1912 gesehen ${ }^{50}$. Er zog einen Text des Altertums ${ }^{51}$ heran, in dem es für das Jahr 673 v. Chr. heißt, «daß die runden Spiegel gar nicht für die heutigen Zwecke der Toilette ersonnen waren, sondern als Schmuck des Gürtels dienten» und verweist auf die Sonnensymbolik der nordeuropäischen Gürtelscheiben. Seine Deutung wird bestätigt durch die Inschrift «ri guang" (= die Sonne scheint) $)^{52}$ des ebenso genannten weit verbreiteten ${ }^{53}$ Spiegeltyps der Han-Zeit, dessen Innendekor durch ein achtfach eingezogenes Mittelfeld gebildet wird ${ }^{54}$.

47 Tilia Tepe (Afghanistan), 1. Jhd. n. Chr. V.I. SARIANIDI, Afganistan : Sokrovitsa besimjannich zarei, Moskau, 1983, fig. 7 - aus Tilia Tepe stammen drei chinesische Spiegel.

48 O.J. MaEnchen-Helfen, Die Welt der Hunnen, Wien-Köln-Graz, 1978 (amerik. 1973), p. 229, 237; LI Xueqin, Eastern Zhou and Qin civilizations, New Haven-London, 1985, p. 296; Japanese Archaeology. History and achievements, Ausstellungskatalog National Museum Tokyo, 1988, ${ }^{\circ}$ 153-155 (je 2 Stück), 157 (6 Stück), 165 (7 Stück), 175 (5 Stück), 212 (6 Stück). Einzelfunde dort sehr häufig.

49 Kaogu (1980) Heft 4, p. 365 (Fig.). Th. LAwTON, Chinese art of the Warring States Period, Freer Gallery Washington, 1982, p. 81. LI, op. cit., p. 297, fig. 126. Auch dieser Spiegel wurde auf der Brust des Toten gefunden. In der Schraffierung der Dreiecke am Rand und der Betonung der Mittelwölbung steht er der spätbronzezeitlichen Goldscheibe von Moordorf (MENGHIN SCHAUER, op. cit., $\mathrm{n}^{\circ} 24$, fig. 63) stilistisch relativ nahe.

50 O. Münsterberg, Chinesische Kunstgeschichte, II, Esslingen, 1912, p. 159.

51 Nach F. HiR'TH, Chinese metallic mirrors, in Franz Boas Anniversary Volume, New York, 1906, p. 208-256.

52 WANG Zhongshu, Han Civilization, New Haven-London, 1982, p. 104, fig. 145.

53 Japanese Archaeology Katalog, op. cit., n 153, 2; 154 B 1; MAENCHEN HELFEN, op. cit., p. 229 sq., fig. 74 (vom Torgun-Fluß im unteren WolgaGebiet). Anm. 167 umschreibt M.-H. den Inhalt der Inschrift mit : «Mögest du die Sonne sehen, die Welt ist strahlend hell».

54 Die Erwähnung der Sonne in der Inschrift und die achtfache Gliederung der Spiegelflache veranlassen mich, eine Vermutung zu wagen: Der heilige Spiegel der japanischen Sonnengöttin und Urahnin des Kaiserhauses, Amaterasu, der - zusammen mit dem siebenarmigen Schwert von Isonokami und dem Halsband des Kaiserpalastes eines der drei «Reichskleinodien» (shintai) - jedem Blick verborgen im Inneren Tempel von Ise bewahrt wird, trägt in den Chroniken der japanischen Frühzeit die Bezeichnung yatakagami, die offensichtlich verschieden interpretiert wird. In den Anmerkungen zum Nihongi, übers. von W.G. AsToN (1896), I, 43, 3, wird er als «eight-hand 
Spiegelscheiben als Grabbeigaben 55 müssen also keineswegs Toilette-Gegenstände sein, haben vielmehr gerade in solchen Fällen, wo die Scheiben auf oder unterhalb der Brust der bestatteten Person gefunden sind ${ }^{56}$, wohl eher eine religiöse Bedeutung gehabt. Da sie in Zentralasien schon sehr früh in sepulkralem Kontext zu finden sind, ist nicht anzunehmen, daß sie erst in mandschurisch-chinesischer Zeit ${ }^{57}$ Bestandteil der neuzeitlichen nordasiatischen Schamanentracht (Abb.

(mirror)» und in denen zum Kojiki, übers. von B.H. CHAMBERLAIN (1882), I, 68, 3 , von älteren japanischen Kommentatoren als «eight heads», «eight feet (long)" oder mit einem "eightfold flowery pattern" je nach Lesung der Zeichen beschrieben. Die Einsetzungsworte, mit denen die Göttin in Gestalt des Spiegels sich offenbart, lauten im Nihongi, I, 83 : «My child, when thou lookest upon this mirror, let it be as if thou wert looking on me. Let it be with thee on thy couch and in thy hall, and let it be to thee a holy mirror». Das liest sich wie eine Umschreibung der Inschrift auf den achtfach gegliederten chinesischen Spiegeln vom Typus ri guang, wie er in den Gräbern der japanischen Kofun-Epoche gefunden wird, Ganz offensichtlich handelt es sich um einen realen Spiegel, denn das Nihongi $(\mathrm{I}, 48)$ bietet sogar die mythische Erklärung für eine kleine Beschädigung, die er aufweist und fügt hinzu : «This is the great Deity worshipped at Ise". J.W. HALL (Das japanische Kaiserreich = Fischer Weltgeschichte, 20, Frankfurt, 1968, p. 39) faßt im Zusammenhang der «drei symbolischen Schätze» des Oberhauptes des Sonnengeschlechts diese theologische Bedeutung mit den Worten : «Von diesen kann der Spiegel als der buchstäbliche 'Körper' der Amaterasu verstanden werden”.

55 In der japanischen Frühzeit ist der Spiegel so eng mit sepulkralen Vorstellungen verbunden, daß eine kaiserliche Prinzessin am Orte, wo sie ihrem Leben aus Gründen der Ehre ein Ende macht, zuerst einen Spiegel im Boden vergräbt (Nihongi, I, 341, für das Jahr 459 n. Chr.). Spiegel gehören dort aber auch andererseits neben Schwert und Schmuck/Halsband zu den drei Gaben mit denen ein Besiegter dem Sieger seine Unterwerfung symbolisiert (zB. Nihongi, I, 220 sq.).

56 Vgl. die hier in Anm. 46, 47 und 49 genannten Bestattungen. Eine Durchsicht der jeweiligen Grabungsberichte dürfte diese Reihe beträchtlich vermehren können. Aus dem neuen komparatistischen Buch von P. LÉvÊQUE (Colère, sexe, rire. Le Japon des mythes anciens, Paris, 1988, p. 113) möchte ich in diesem Kontext Worte aus dem Kojiki, die er als zen-buddhistisch gefärbte Anweisung erkennt, entnehmen : «Respirez l'esprit du Soleil, placez-le à hauteur du ventre. Lorsqu'on pratique ainsi pendant des années, le bas-ventre devient dur comme le métal ou la pierre. Sinon, on ne peut prétendre être un homme de la Voie». - Dem Atmen hier entspricht übrigens ebenso der Vorgang, in dem der sonnenhafte Branchos von Apoll mit mantischer Begabung angehaucht (epipnous) wird (KoNON, Narr., 33, 4) wie die Anweisungen der Mithras-Liturgie, von den Strahlen der Sonne Atem zu holen (hier Anm. 43, v. 538, 630).

57 So EliADE, op. cit., p. 156. 
$4)^{58}$ geworden sind. Ich vermute, daß sie dort viel älter sind, ihre Spiegelfunktion in Dämonenbeschwörungen aber erst sekundär ist, denn in den tibetischen 'Chams-Tänzen tragen auch die jeweils dargestellten Gottheiten eine me-long genannte runde Scheibe auf der Brust "which is absolutely necessary", wie es im 'Chams yig heißt ${ }^{59}$. Sonnensymbolik dürfte die ursprüngliche Bedeutung der Schamanenspiegel sein.

Vergleiche über große zeitliche und räumliche Distanzen scheinen mir methodisch nicht nur unbedenklich, sondern angesichts der Parzellierung des modernen Wissenschaftsbetriebes dringend geboten, da sie von den Quellen und den Fragestellungen gefordert werden. In zwei schon erwähnten Beispielen - der Langlebigkeit des Motivs der geflügelten Sonnenscheibe ${ }^{60}$ und dem Auftreten des Tierkampfes in der Mongolei und in Syrien 61 - wird dies besonders sinnfällig. Hinzufügen möchte ich noch den Hinweis auf das erstaunlich hohe Alter des Motivs der neuzeitlichen tibetischen Tiger-Teppiche. Es ist sehr viel älter als die Tibetologie annimmt ${ }^{62}$. Denn sie haben ihre exakten Entsprechungen in den Stickereien aus dem 6. Kurgan von Noin Ula (1. Jhd. v. Chr.) ${ }^{63}$. Die Art der Tigerfell-Streifen in geschwungenen Doppellinien findet sich schon einige Jahrhunderte

58 W. Heissig - C.C. MÜLLER (ed.), Die Mongolen. Ausstellungskatalog München und Hildesheim, I, Innsbruck-Frankfurt, 1989, $n^{\circ} 165$, fig. p. 148; $n^{\circ} 166$, fig. p. 153. Die zweite Schamanenfigur hat den Spiegel knapp unter dem Hals umgehängt. Dort trägt auch die von Strahlen umgebene Gestalt mit der Lanze als Auferstehungssymbol auf dem fränkischen Bildstein von Niederdollendorf einen «scheibenartigen Gegenstand» (Reallex. German. Altert., $\mathrm{II}^{2}$, p. 555, pl. 45c - H. RotH. Cf. METZLER, in Visible Religion, 7 (1990), n. 72, im Druck). Wohl kaum ein Christus als Triumphator !, cf. vielmehr HALIFAX (supra n. 23), p. 90 sq. - C.C. Müller, in A. EgGEBRECHT (ed.), Die Mongolen und ihr Weltreich, Mainz, 1989, p. 174 : neun Spiegel üblich.

59 R. v. NEBESKY-WoJKOWITZ, Tibetan religious dances. Text and translation of the 'Chams yig, den Haag-Paris, 1976, p. 95, 72, 115. Darstellungen von 'Chams-Figuren mit Spiegeln auf der Brust zB. bei HEISSIG-MÜLLER, op. cit., II, p. 240-243.

60 MAYER-OPIFICIUS, cf. supra n. 38.

61 ALTHEIM, cf. supra n. 24.

62 J. \& B. FORD, in LiPTON, op. cit. n. 32, p. 160 sq.

63 G. BOROFFKA, Wanderungen eines archaisch-griechischen Motives über Skythien und Baktrien nach Alt-China, in Festschrift 25 Jahre Röm. German. Kommission 1927, Berlin, 1930, p. 52-81, bes. 34-38; S.I. RUDENKo, Die Kulturen der Hsiung-nu und die Hügelgräber von Noin Ula, Bonn, 1969 (russ. 1962), p. 76 sq., pl. 49-50. 
früher ebenfalls in der Mongolei auf einer Goldplatte mit Tierkampfszene ${ }^{64}$.

Doch zurück in die nähere zeitliche und räumliche Umgebung des Mopsos auf den Münzen von Mallos : Auf den Orthostaten von Karatepe findet sich die, wie es scheint ungedeutete Darstellung eines Mannes im Knielauf-Schema zwischen zwei Reitern, deren Pferde er an den Zügeln hält ${ }^{65}$. Dieser Mann trägt nach Autopsie - Photographieren war auch mehr als vierzig Jahre nach der Ausgrabung noch verboten ! eine große runde Scheibe vor der Brust, die wegen der Tragweise nicht als Schild zu erklären ist, vielmehr an die Scheibe in den Händen der Flügelgestalt auf den Münzen erinnert. Der Dargestellte könnte also Mopsos als Anführer verschiedener Gruppen seiner Leute (siehe unten s. 247) oder als Versöhner (diallaktés) bei der Gründung von betMupšu/Mopsuhestia oder eben von Mallos sein. Denkbar ist allerdings auch die umgekehrte Erklärung: Mopsos mit der Sonnenscheibe nach dem Bild des einstweilen noch Unbekannten in Karatepe geformt!

In Karatepe wurde bekanntlich 1946 von H.Th. Bossert die bilingue Inschrift des Königs Asitawadas gefunden 66 , in der das "Haus des Mpš/Mukšuš” erwähnt wird - Haus oder ${ }^{67}$ Dynastie jenes Mukšuš, den auch die ältere, schon länger bekannte Inschrift des Königs Madduwattaš nennt ${ }^{68}$. Mukšuš wurde zuerst von E. Forrer mit dem Mopsos/Moxos der griechischen Tradition identifiziert ${ }^{69}$. Sein Name ist einerseits im luwischen Sprachgebiet Anatoliens ${ }^{70}$ gut belegt, andererseits begegnet er auch schon in den frühgriechischen Linear-B Texten sowie in Thessalien und in Abwandlungen auch in Attika ${ }^{71}$.

64 LI, op. cit. n. 48, p. 333 sq., fig. 149, Fundort : Xigoupan im Zhungor Banner. Boroffkas These eines westlichen Einflusses wird davon natürlich nicht berührt.

65 P. MATTHIAE, Studi sui rilievi di Karatepe, Rom, 1963, p. 58, 108 sq., pl. VI (SOI-NO-4).

66 H.Th. BosserT, Karatepe. Erster Vorbericht, Ankara, 1950, p. 62.

67 Zur Problematik der genealogischen oder topographischen Lesung von "Haus» : J. OBERMANN, Discoveries at Karatepe. A phenician royal inscription from Cilicia, New Haven, 1948, p. 19, 38-40.

68 A. GoETzE, Maduwattaš, Leipzig, 1928 (Mitteil. der Vorderasiat.-ägypt. Gesellsch., 32, 1), p. 37.

69 E. FORRER, in Klio, 30 (1937), p. 177.

70 Ph.H. HoUwINK TEN CATE, The Luwian population groups of Lycia and Cilicia Aspera during the hellenistic period, Leiden, 1965, p. 44-50; L. ZGUSTA, Kleinasiatische Personennamen, Prag, 1964, p. 960.

71 Linear B : KN De 1381 und PY Sa 774. RosCHER und RE, art. Mopsos. 
Und natürlich gibt ihm der griechische Mythos auch eine griechische Genealogie: Mopsos ist Sohn der Manto, der Tochter des Sehers Teiresias, als sein Vater wird wie oft bei Sehern/Schamanen der Gott Apollo ${ }^{72}$ selbst genannt - eine ziemlich durchsichtige Konstruktion also, die mit redenden Namen die Begabung des Schamanen zu begründen versucht ${ }^{73}$. Das gilt übrigens auch für den thessalischen Mopsos. Der Name seines Vaters ist nämlich auch Lebes ${ }^{74}$ (= Kessel), spielt also auf schamanistische Zerstückelungs- und Auferstehungsrituale nach Art des sogenannten Medeafrevels ${ }^{75}$ oder der Bestattung der tibetischen Könige ${ }^{76}$ an.

Wie der Name Mopsos also sinnvoll in der historischen Überlieferung Kilikiens verankert ist, so weist auch der Name des Kalchas, der nach einigen antiken Autoren nicht nur in Klaros mit Mopsos konfrontiert ist, sondern als Kolonistenführer und Seher auch in Kilikien tätig ist, in dieses Gebiet ${ }^{77}$. Denn «Kalchas» kann durch Hauchversetzung 78 aus Chalkas mit der alten Namensform des Landes $=$ Hilakkai ${ }^{79}$ verbunden werden, die ihrerseits wie I. Lévy überzeugend

72 Vgl. den Seher Seilenos, Sohn eines «pontischen Weibes» (PLUT., Lysandr., 26, 1) oder den thrakischen König Kotys (Inschrift auf der Kanne $n^{\circ} 112$ des Schatzfundes von Rogozen/Bulgarien).

73 W. BURKERT, Die orientalisierende Epoche in der griechischen Religion und Literatur, Heidelberg, 1984 (Sitz.ber. Akad. Wiss.), p. 54.

74 H.W. PARKE, The oracles of Apollo in Asia Minor, London, 1985, p. 243, n. 10. Cf. H.S. VERSNEL, Apollo and Mars one hundred years after Roscher, in Visible Religion, 4-5 (1985-1986), p. 158, n. 104, mit Hinweis auf OGIS 611; F. GRAF, Nordionische Kulte, Roma, 1985, p. 320 sq. zum kraterismós.- Cf. auch A.F. LAURENS, L'enfant entre l'épée et le chaudron, in $D H A, 10$ (1984), p. 203252 , bes. 228 (freundlicher Hinweis von A. Verbanck-Piérart).

75 W. Burkert, Homo necans, Berlin-New York, 1972, p. 50 sq., 97 sq. Bemerkenswerterweise gibt es eine Reihe von Vasenbildern, die den positiven Ausgang des Rituals zeigen : K. STÄHLER, Eine Sammlung griechischer Vasen - J.D. in Ostwestfalen, Münster, 1983, $\mathrm{n}^{\circ} 22$ mit Literatur; LAURENS, art. cit., fig. 23, 26.

76 E. HAARH, The Yar-luñd dynasty, Kobenhavn, 1969, p. 348 sq.

77 H. GRégoIRe, A propos de Karatepe : Mopsos et Calchas, in NClio, 1-2 (19491950), p. 162-165.

78 H. FRISK, Griechisches etymologisches Wörterbuch, I, Heidelberg, 19732, p. 769, s.v. kalche.

79 H. GRÉGOIRE, art. cit., p. 164 sq. 
nachweisen konnte, als Hylachaioi hinter den von Herodot mißverstandenen Hypachaioi zu sehen sind ${ }^{80}$.

Wie Kalchas um 400 v. Chr. als geflügelter Seher dargestellt werden konnte, so glaube ich auch Mopsos in diesem Typus nachgewiesen zu haben. Um dieses ikonographische Problem geht es hier, nicht um die traditionskritische Analyse der einzelnen Überlieferungsstränge über die verschiedenen Träger dieses Namen in den griechischen Texten 81 und auch nicht um die Historizität ${ }^{82}$ der den Mythen zugrundeliegenden Gestalt. Da dazu einschlägige Darstellungen vorliegen, hier nur einige ergänzende Beobachtungen, die insofern generalisierender Art sind, als die griechischen Berichte über den in orientalischen Quellen erwähnten, aber über die räumliche und zeitliche Ansetzung in Kilikien am Ende des 2. Jahrtausends v. Chr. hinaus nicht näher charakterisierten Mopsos möglicherweise auch nur Typenspezifisches aussagen. Das läßt sich vereinfachend mit den Aspekten seines Gottes und Vaters Apollo umschreiben.

Wie dieser ist Mopsos Seher, Heiler, Krieger und - so möchte ich vorschlagen - über den Strahlendiskus auf der Münze mit der Sonne verbunden. Während seine mantischen Funktionen schon seit dem 7 . Jhd. v. Chr. von Kallinos von Ephesos ${ }^{83}$ erwähnt werden, ist seine Verehrung als "Arzt der Menschen" einstweilen erst durch eine Inschrift der Kaiserzeit belegt ${ }^{84}$. Was das Kriegertum betrifft, so hat erst jüngst H.S. Versnel in seiner Untersuchung über Apollo und Mars auf die engen Verbindung beider Gottheiten mit den Aussendungsritualen vom Typus ver sacrum und apoikía toû theôे hingewiesen ${ }^{85}$. R.D. Barnett nennt den Mukšuš der Inschrift des Madduwattaš "Freibeuter»86. Pausanias' Nacherzählung der kolophonischen Traditionen 87 über die an der Gründung von Klaros beteiligten Kriegergruppen mit ihrer Betonung von Umherziehen und

80 I. LÉvy, Hylachaioi, in Mélanges E. Boisacq, 2 = Ann. de l'Inst. de Phil. et d'Hist. Orient. et Slave, 6 (1938), p. 119-127 - nach GRÉGOIRE, art. cit., p. 165.

81 ImMISCH, cf. supra n. 41.

82 R.D. BARNETT, Mopsos and the Dnnym, in $C A H$, II, $2(1975)^{2}$, p. 363-366, N.G.L. HAMMOND, ebd. 679 sq.

83 KALLINOS, fr. 8 = STRAB., XIV, 4, 3 (668).

84 E. Doblhofer, Mopsos Arzt der Menschen, in ÖJH, 46 (1961-1963), Beilage 514. G. DAGRoN - D. FeIsSeL, Inscriptions de Cilicie, Paris, 1987, p. 134.

85 VERSNEL, art. cit., p. 140 sq.

86 BARNETT, art. cit., p. 363.

87 PAUS., VII, 3, 1-2. 
Gewalttätigkeit läßt diese Charakterisierung berechtigt erscheinen. Genannt werden vom Hellenentum zunächst "Kreter" und dann der "Haufen (plêthos), der Rhakios folgte (heipeto)». Dieser trifft auf Manto und macht sie und «die, die mit ihr ziehen, zu Mitsiedlern (sýnoikoi). Rhakios wird zwar der sterbliche Vater des Mopsos, Apollo aber hat höhere Rechte über Manto, denn dem Gotte war sie als Kriegsgefangene nach Delphi geweiht worden, und als Besitz des Gottes war sie mit einer apoikia nach Asien geschickt worden. Beider Sohn Mopsos vertrieb die um Klaros wohnenden Karer endgültig aus ihrem Land.

Gegenüber seinen Gefolgschaften ${ }^{88}$ muß sich Mopsos offensichtlich durch seine Führerqualitäten legitimieren und diese gegenüber Konkurrenten in Zweikämpfen verteidigen. Kalchas besiegt er im unblutigen Schamanenwettstreit, der Kampf mit Amphilochos bei Mallos endet für beide tödlich ${ }^{89}$. Mantische und politisch-militärische Autorität sind hier vereint wie mehrfach bei Sehern von Elis 90 , beim frührömischen König, der verkündet, was ihm auf dem Wege der Inspiration als Wille des Gottes» ( $f a s$ ) offenbart worden ist ${ }^{91}$, bei dem thrakischen «Priester und Heerführer» Kosingas 92 oder dem awarischen Schamanen und Heerführer Bookolabras ${ }^{93}$. Vergleichbar scheinen mir in Kleinasien auch die Gruppen der Korybanten, insofern man sie als männerbündische Ekstatiker begreift, denn zwei Korybanten werden auch als Gründer von Städten genannt : Akmon in Akmonia und Prymmneus in Prymnessos ${ }^{94}$ - durchsichtige Konstruktionen wiederum, aber ausdrücklich Korybanten. Als Tänzer (mólpoi) geehrt bilden ihnen vergleichbare Kriegergruppen in Milet seit dem 6. Jahrhundert faßbar die Elite der Stadtbürger ${ }^{95}$.

88 S. Deger-JalKotzKy, E-QE-TA. Zur Rolle des Gefolgschaftswesens in den Mykenischen Reichen, Wien, 1978.

89 STRAB., XIV, 1, 27 (624-643); 5, 16 (676).

90 F. MORA, Gli indovini elei e la guerra, in CISA, 10 (1984), p. 31-40 (freundlicher Hinweis von G. Casadio).

91 U. v. LÜвTOW, Recht und Rechtswissenschaft im Rom der Frühzeit, in Festschrift für G. Radke, Münster, 1986, p. 164, 166.

92 Polyainos, VII, 22. MeUli, art. cit., p. 163, n. 3.

93 Theophylaktos SimoKatTes, 53, 14-19. G. MoRAvcsiK, Byzantinoturcica, II, Berlin DDR, 1958, p. 95.

94 STRUBBE, art. cit. n. 1, p. 259.

95 S. LURIJA, Ein milesischer Männerbund im Lichte ethnologischer Parallelen, in Philologus, 83 (1928), p. 113-136, bes. 113, 119; H. JeanmaIre, Couroi et Couretes, Lille, 1939, p. 550; METZLER, art. cit. n. 6, p. 78, n. 77. 
$\mathrm{Zu}$ Erwägungen über die mutmaßliche Zusammensetzung der Gefolgschaften gibt der Name der Landschaft Pamphylien Anlaß, die nach Plinius $(N H, \mathrm{~V}, 96)$ früher Mopsopia geheißen habe, was insofern in den chronologischen Kontext paßt, als der pamphylische Dialekt sehr alte, vordorische Elemente des peloponnesischen Griechisch bewahrt hat ${ }^{96}$ und damit übrigens auch den Personennamen Mo-qo-so in Linear B-Texten sinnfällig werden läßt. Denn wie die Leute des Rhakios verschiedener Herkunft waren, so meint ja auch nach griechischer Deutung Pam-phylien die Zusammensetzung seiner Bevölkerung aus allen (möglichen) Stämmen. Das kann so zu verstehen sein wie das Panellênon oizýs = das Elend ganz Griechenlands, wie Archilochos (fr. 54) sich und seine Mitsiedler auf Thasos sieht - pejorativ gemeint wie die $k a k o \hat{\imath}$, die laut Konon ${ }^{97}$ Jonien und Äolien neu besiedelt hatten, eine colluvies gentium ${ }^{98}$ also, die zu politischer Gruppenbildung geführt hat. Im Falle Pamphyliens folgten sie zusammen mit den Leuten des Kalchas ${ }^{99}$ dem Mopsos, der dort ja auch als Gründer von Aspendos, Phaselis, Perge und Syllion 100 verehrt wurde. Die Frage, ob die historischen Prototypen ihrer jeweiligen mythischen Berichte Griechen oder Anatolier waren, kann mit dem Hinweis auf die gerade auch durch den Namen Pam-phylien symbolisierte Komplexität der Völkerenstehung - in diese frühe Phase weisen ja die Berichte über die Koloniegründer - offen bleiben, denn durch ihre Darstellung als Flügelwesen waren die Seher Mopsos und Kalchas im Bewußtsein ihrer antiken Nachwelt schamanischen Leitbildern entsprechend ohnehin als höhere Wesen charakterisiert.

Universitat - FB 10

Dieter METZLER

Pferdegasse, 3

D - 4400 MÜNSTER

96 S.C. BRIXHe, Le dialecte grec de Pamphylie. Documents et grammaire, Paris, 1976, p. 145 sq.

97 KoNoN, Narr., $29=26$ F $1,29 \mathrm{~J}$.

98 R. WENskUs, Stammesbildung und Verfassung. Das Werden der frühmittelalterlichen gentes, Köln-Graz, 1961, p. 449 sq.

99 PAUS., VII, 3, 7. Cf. IMMISCH, art. cit. n. 2, p. 162-164.

100 MAGIE, op. cit. n. 2; D. HEREWARD, Inscriptions from Pamphylia and Isauria, in JHS, $78(1958)$, p. $57-77$, bes. 57 sq. 


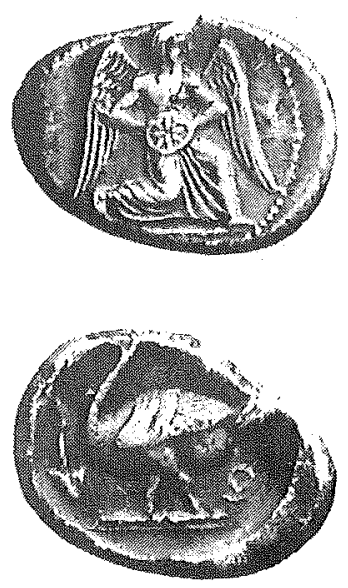

Abb. 1

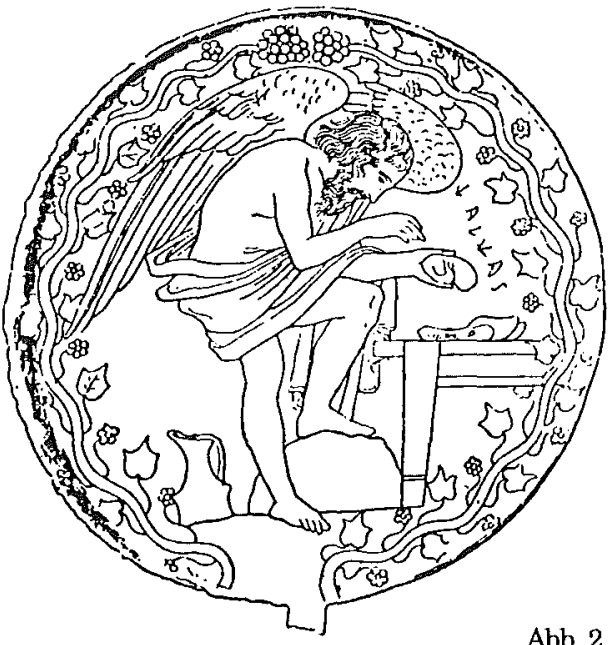

Abb. 1: Mopsos auf einem Stater von Mallos (Photo Münzen und Medaillen AG) Abb. 2: Kalchas auf einem etruskischen Bronzespiegel (nach E. Gerhard)
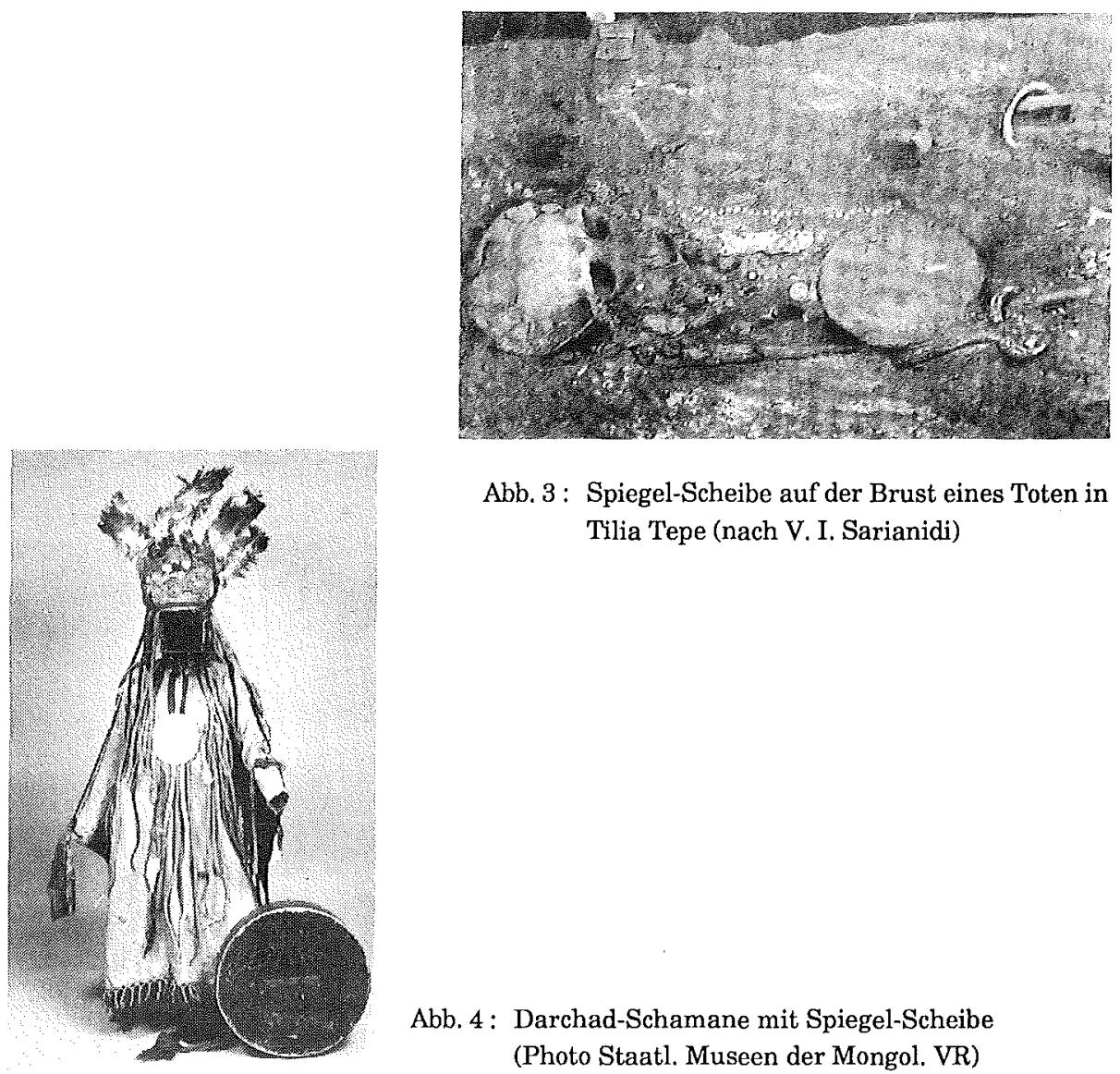

Abb. 3: Spiegel-Scheibe auf der Brust eines Toten in Tilia Tepe (nach V. I. Sarianidi)

Abb. 4: Darchad-Schamane mit Spiegel-Scheibe (Photo Staatl. Museen der Mongol. VR) 